\title{
Discordant Pacing Manoeuvers and a Narrow QRS Tachycardia - What is the Tachycardia Mechanism?
}

\author{
Hussam Ali, ${ }^{1}$ Pierpaolo Lupo, ${ }^{1}$ Sara Foresti, ${ }^{1}$ Guido De Ambroggi, ${ }^{1}$ Gianluca Epicoco ${ }^{1}$ and Riccardo Cappato ${ }^{1,2}$ \\ 1. Arrhythmia \& Electrophysiology Unit II, Humanitas Gavazzeni Clinics, Bergamo, Italy; 2. Arrhythmia \& Electrophysiology Research Center, IRCCS Humanitas
}

Research Hospital, Rozzano (Milan), Italy

\begin{abstract}
A 24-year-old female underwent an electrophysiological study because of recurrent episodes of drug-refractory, paroxysmal supraventricular tachycardia. During adrenergic stress, a narrow QRS tachycardia with eccentric atrial activation was reproducibly inducible. The response to premature ventricular extrastimulation during tachycardia suggested the presence of a slowly conducting accessory pathway. However, a comprehensive appraisal of the electrophysiological study delineated the tachycardia substrate as an atypical atrioventricular nodal reentrant tachycardia in the presence of a bystander nodofascicular pathway. Careful analysing of the basal pacing manoeuvers during sinus rhythm (para-Hisian and differential ventricular pacing) was crucial to establish the correct diagnosis and to avoid unnecessary left-side access to ablate this tachycardia.
\end{abstract}

\section{Keywords}

Narrow QRS tachycardia, para-Hisian pacing, atypical AVNRT, nodofascicular pathway

Disclosure: Hussam Ali, Pierpaolo Lupo, Sara Foresti, Guido De Ambroggi, Gianluca Epicoco and Riccardo Cappato have no conflicts of interest to declare. Compliance with Ethics Guidelines: Informed consent for the procedure was obtained from the patient.

Open Access: This article is published under the Creative Commons Attribution Noncommercial License, which permits any non-commercial use, distribution, adaptation and reproduction provided the original author(s) and source are given appropriate credit.

Received: 27 June 2015 Accepted: 20 July 2015 Citation: European Journal of Arrhythmia \& Electrophysiology, 2015;1(1):32-4 DOI: 10.17925/EJAE.2015.01.01.32

Correspondence: Hussam Ali, Arrhythmia \& Electrophysiology Unit II, Humanitas Gavazzeni Clinics, Via M. Gavazzeni 21, 24125 Bergamo, Italy. E: hussamali.ep@gmail.com

\section{Case Presentation}

This case concerns a 24-year-old female with recurrent episodes of paroxysmal palpitations. Her physical exam, 12-lead electrocardiogram (ECG), 24-hour Holter monitoring and echocardiogram did not reveal any abnormality. In the last 2 years, she had several admissions to the emergency department where paroxysmal, adenosine responsive, supraventricular tachycardia was documented. Anti-arrhythmic drug therapy (beta-blocker, flecainide) was ineffective, and the patient was referred to our centre for electrophysiological evaluation.

After obtaining informed consent, an electrophysiological study was performed under conscious sedation. Multipolar diagnostic catheters were positioned in the coronary sinus (CS), His bundle (HB) region and right ventricle (RV). Baseline atrio-His (AH) and His-ventricular (HV) intervals were 60 and 55 ms, respectively. RV pacing showed slow, decremental and eccentric ventriculoatrial (VA) conduction (earliest atrial activation at the level of mid-CS). The response to para-Hisian pacing manoeuver is shown in Figure 1.

During atrial pacing, there was no evidence of ventricular preexcitation or antegrade dual atrioventricular (AV) node physiology. No tachycardia was inducible at the baseline state.

After isoproterenol infusion ( $2 \mathrm{mcg} /$ minute), and with aggressive atrial burst pacing (240 ms), a narrow QRS tachycardia was reproducibly inducible. Tachycardia cycle length had an average of 320 ms; AH and
HA intervals were 75 and 245 ms, respectively. Notably, tachycardia induction was independent of critical prolongation in the AH interval.

Figure 2 demonstrates ECG and intracardiac recordings during tachycardia and the effect of a premature ventricular extrastimulus (PVE) $\left(\mathrm{S}_{2}\right)$. Based on these data, what is the most likely mechanism of this tachycardia?

\section{Discussion}

The retrograde conduction pattern (slow-decremental-eccentric) raised the suspicion of a left-sided, Coumel-type accessory pathway (AP). However, eccentric retrograde conduction over the nodal slow pathway (SP), particularly over the left posterior extension, has been described. ${ }^{1}$ The para-Hisian pacing manoeuver is helpful when elucidating the retrograde conduction pattern and to explore the presence of occult APS. $^{2}$ In Figure 1, the loss of direct HB capture was associated with a significant increase in the stimulus-atrium (Stim-A) intervals without any change in retrograde atrial activation sequence (RAAS). This response confirms that retrograde conduction is His-dependent (i.e., nodal conduction) excluding an extranodal, septal, AP. Although this manoeuver may have limited utility with slowly conducting APS, this is usually due to the dominate retrograde conduction over the nodal fast pathway. In this case, retrograde conduction was constantly slow with long VA intervals (i.e., no retrograde conduction over the fast pathway), making such a masquerading effect unlikely. Additionally, retrograde conduction over a nodo-fascicular pathway (NFP) may still give a nodal response during this manoeuver because of its direct connection to the 
His-Purkinje system. In this patient, another manoeuver was performed to exclude the presence of an occult left-sided AP, by pacing the basal left ventricle (LV) through a posterior branch of the CS (see Figure 3).

This manoeuver was performed during isoproterenol infusion and stable VA retrograde conduction, and with the tachycardia was easily inducible. The differential ventricular pacing, at the same pacing cycle length (400 ms), showed longer Stim-A and local VA intervals during LV pacing (280 ms and $220 \mathrm{~ms}$ ) compared with that during RV pacing (220 and $102 \mathrm{~ms})$, respectively. This makes the presence of a left-sided AP unlikely.

Alternatively, differential RV apex/base pacing could be also used to differentiate the retrograde conduction pattern. ${ }^{3}$ The differential diagnosis of the induced tachycardia (AH > HA interval) basically includes: orthodromic AV reentrant tachycardia (O-AVRT) using a slowly conducting AP (permanent junctional reciprocating tachycardia (PJRT) 'Coumel tachycardia'); atypical (fast-slow) AV nodal reentrant tachycardia (AVNRT); and atrial tachycardia.

The RAAS during tachycardia was identical to that obtained during RV and LV pacing making the diagnosis of AT unlikely, but still possible. The response to PVE (see Figure 2), reproducibly delayed the next atrial activation without altering antegrade HB activation. This phenomenon confirms the presence of an AP bypassing the HB and excludes the diagnosis of AT. This paradoxical delay in atrial activation in response to PVE could be observed during PJRT using a slowly conducting AV bypass. However, the response to para-Hisian pacing and differential LV-RV pacing showing identical RAAS to that during tachycardia favours the diagnosis of an occult NFP connected to the left extension of the nodal SP.

Accordingly, the presented tachycardia could be an O-AVRT using an NFP for the retrograde limb, or an atypical fast-slow AVNRT with a bystander occult NFP. During AVNRT, a His-refractory PVE should not have any effect on the ongoing tachycardia. However, the presence of an NFP connected to the nodal SP may explain the paradoxical delay with $\mathrm{S}_{2}$ (see Figure 2) maintaining the same RAAS.

In order to elucidate the role of this NFP in tachycardia mechanism, a right bundle branch block (RBBB) was intentionally induced by applying gentle pressure against the early proximal portion of the RBB. The induced proximal RBBB did not affect the ongoing tachycardia cycle length, and the His-atria (HA) intervals remained unchanged (245 ms) (see Figure 4). The latter phenomenon confirmed the bystander role of the NFP in this case. An O-AVRT using a right-sided NFP typically shows significant tachycardia slowing when RBBB develops. ${ }^{4}$ Importantly, the longer VA interval during LV pacing compared with that during RV pacing points against the presence of a left-sided NFP.

Finally, based on these electrophysiological features and phenomena, the best explanation of tachycardia mechanism is atypical AVNRT with a bystander occult NFP. This NFP inserted into the left nodal SP provides the route for PVE during His refractoriness to penetrate and prolong the refractoriness and conduction over the nodal SP during tachycardia.

Ventricular entrainment was not performed in this case that might have added further evidence of tachycardia mechanism through analysing RAAS, AV response after pacing termination and the post-pacing interval.

Conventional mapping during tachycardia revealed a relatively late activation at the right interatrial septum adjacent to the CS ostium.

\section{Figure 1: Para-Hisian Pacing Manoeuver}



At a low pacing output, there was a loss of direct His bundle (HB) capture at the second beat as indicated by a wider QRS morphology and retrograde activation of HB (the grey arrow). This delay in HB activation was associated with a significant increase in the stimulus-atrium (Stim-A) interval (from $250 \mathrm{~ms}$ to $300 \mathrm{~ms}$ ). The asterisks indicate the site of earliest retrograde atrial activation at the level of the mid-coronary sinus (CS) (CS 5-6) as illustrated by the fluoroscopic image (left anterior oblique [LAO] $30^{\circ}$ ) at the right side.

\section{Figure 2: Surface ECG $\left(I, a V F, V_{1}, V_{5}\right)$ and Intracardiac Recordings during Tachycardia and the Effect of His-synchronous PVE $\left(\mathrm{S}_{2}\right)$}

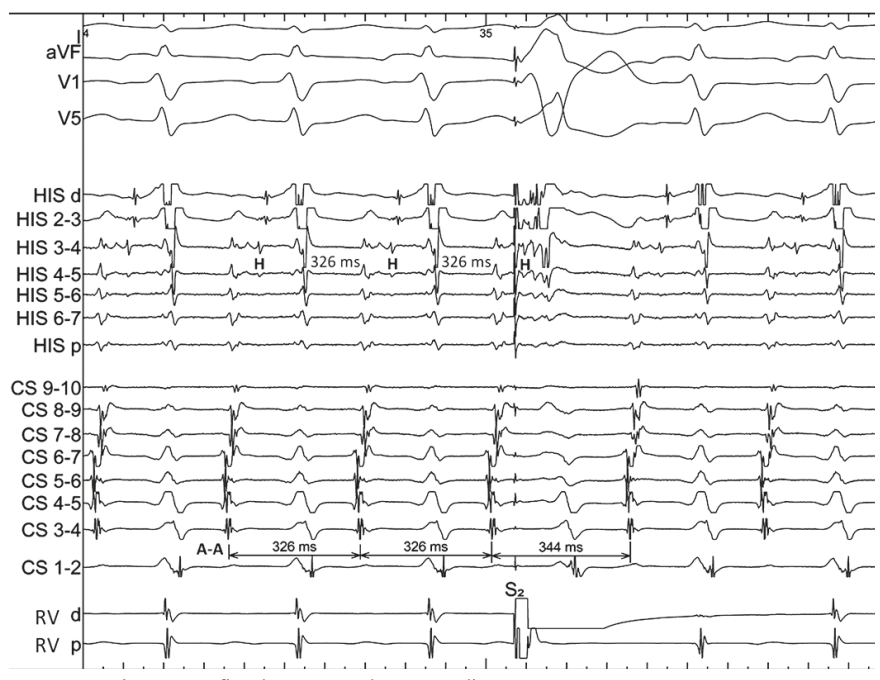

aVF = arteriovenous fistula; $E C G=$ electrocardiogram; $P V E=$ premature ventricular extrastimulus.

\section{Figure 3: Differential Ventricular Pacing from the RV (Left) and LV (Right)}
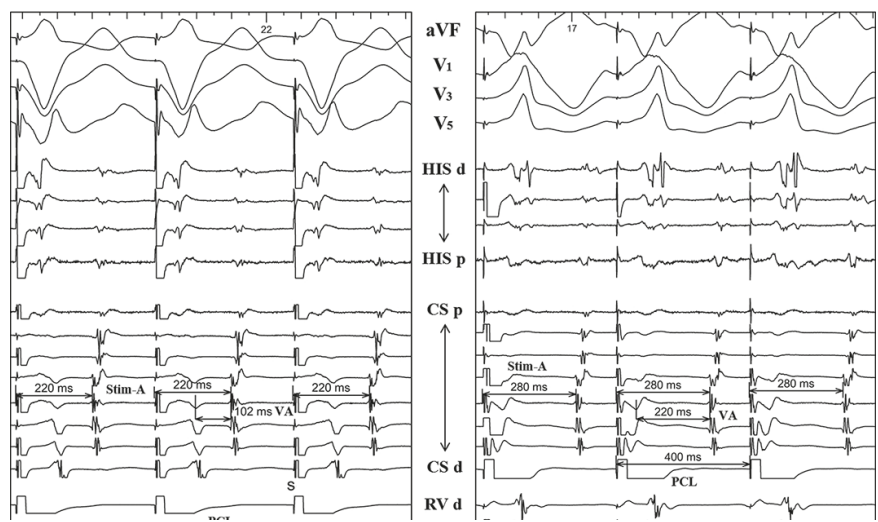

$L V=$ left ventricle; $P C L=$ paced cycle length; $R V=$ right ventricle; Stim- $A=$ stimulus atrium; $V A=$ ventriculoatrial. 


\section{Figure 4: 12-lead ECG and His Bundle Recordings during Tachycardia (Panel A), and after the Mechanically Induced RBBB (Panel B)}
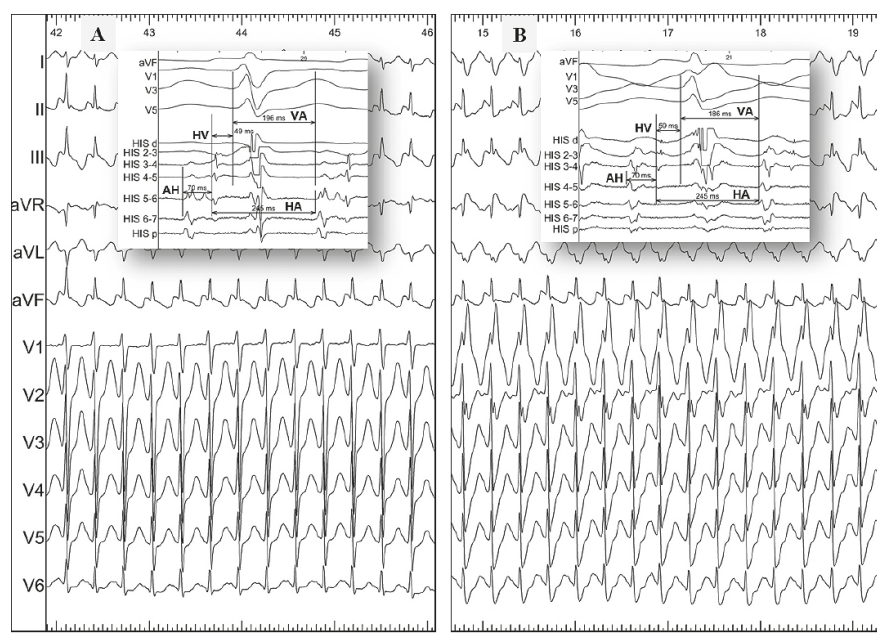

$E C G=$ electrocardiogram; RBBB = right bundle branch block.
However, standard, right-sided, RF ablation of the nodal SP at the area between the CS ostium and the tricuspid valve rendered the tachycardia non-inducible. The patient remained asymptomatic, off anti-arrhythmic drugs, during an 18-month follow-up period.

Even if there is no conclusive evidence, the successful RF at an area relatively distant from the earliest activation site during tachycardia is in favour of atypical AVNRT rather than PJRT using a slowly conducting AV bypass tract.

This case highlights the crucial role of basal electrophysiological study to fully understand the tachycardia mechanism. In this case, analysing only tachycardia features with eccentric RAAS and the response to PVE, would have led to the diagnosis of O-AVRT and an unnecessary left side access to map and ablate the earliest atrial site. Careful analysis of basal pacing manoeuvers (para-Hisian and differential RV/LV pacing) was critical to delineate the tachycardia substrate. Furthermore, mechanical RBBB might be induced intentionally for diagnostic utility in exceptional cases. Such cases may be rare and conclusions cannot be generalised, even so familiarity with different pacing manoeuvers may help to understand arrhythmia mechanisms and to face challenging cases.
1. Otomo K, Nagata Y, Uno K, et al., Atypical atrioventricular nodal reentrant tachycardia with eccentric coronary sinus activation: electrophysiological characteristics and essential effects of left-sided ablation inside the coronary sinus, Heart Rhythm, 2007;4:421-32.
2. Hirao $K$, Otomo $K$, Wang $X$, et al., Para-Hisian pacing. A new method for differentiating retrograde conduction over an accessory AV pathway from conduction over the AV node Circulation, 1996;94:1027-35.

3. Martínez-Alday JD, Almendral J, Arenal A, et al., Identification of concealed posteroseptal Kent pathways by comparison of ventriculoatrial intervals from apical and posterobasal right ventricular sites, Circulation, 1994;89:1060-7.

4. Ho RT, Pavri BB, A long RP-interval tachycardia: What is the mechanism?, Heart Rhythm, 2013;10:456-8. 